\title{
Hospital Utilization, Costs, and Mortality for Adults With Multiple Chronic Conditions, Nationwide Inpatient Sample, 2009
}

Claudia A. Steiner, MD, MPH; Bernard Friedman, PhD

Suggested citation for this article: Steiner CA, Friedman B. Hospital Utilization, Costs, and Mortality for Adults With Multiple Chronic Conditions, Nationwide Inpatient Sample, 2009. [Erratum appears in Prev Chronic Dis $2013 ; 10$. http://www.cdc.gov/pcd/issues/2013/12_0292e.htm.] Prev Chronic Dis 2013;10:120292. DOI: http://dx.doi.org/10.5888/pcd10.120292 国.

PEER REVIEWED

\section{Abstract}

\section{Objective}

Our objective was to provide a national estimate across all payers of the distribution and cost of selected chronic conditions for hospitalized adults in 2009, stratified by demographic characteristics.

\section{Analysis}

We analyzed the Nationwide Inpatient Sample (NIS), the largest all-payer inpatient database in the United States. Use, cost, and mortality estimates across payer, age, sex, and race/ethnicity are produced for grouped or multiple chronic conditions (MCC). The 5 most common dyads and triads were determined.

\section{Results}

In 2009, there were approximately 28 million adult discharges from US hospitals other than those related to pregnancy and maternity; $39 \%$ had 2 to $3 \mathrm{MCC}$, and $33 \%$ had 4 or more. A higher number of MCC was associated with higher mortality, use of services, and average cost. The percentages of Medicaid, privately insured patients, and ethnic/racial groups with 4 or more MCC were highly sensitive to age.

\section{Summary}

This descriptive analysis of multipayer inpatient data provides a robust national view of the substantial use and costs among adults hospitalized with MCC.

\section{Introduction}

The prevalence of adults with more than 1 coexisting chronic condition, often referred to as multiple chronic conditions (MCC), is large and growing in the United States. As the US population ages, the Partnership for Solutions projects that by 2020, one-quarter of Americans will live with MCC (1). Consequences of multiple chronic conditions include impacts on health, quality, delivery of care, and cost. Nationwide, expenses for hospital inpatient care remain the largest component of total health care expenditures. A previous study of hospitalizations using a national all-payer database demonstrated that the number of chronic conditions independently influences hospital costs. Patients with complex illness, defined as 3 or more chronic conditions, were found to have a disproportionately large association with hospital cost per year (2). Another study using hospitalizations from statewide databases that support readmission analyses demonstrated that the likelihood of a readmission was related to the complexity of chronic illness as measured by the number of different chronic conditions (3).

The primary objective of this study is to describe the distribution of multiple chronic conditions among patients hospitalized in US community hospitals in 2009. The study includes all payers, including private, public, and uninsured. We also describe hospitalizations subclassified by patient's payer or by race/ethnicity, within age groups 
and by sex. In addition, we describe the most common dyads and triads of chronic conditions by demographic characteristics.

\section{Analysis}

The data source for this study is the Nationwide Inpatient Sample (NIS) of the Healthcare Cost and Utilization Project (HCUP), the largest all-payer inpatient database in the United States (4). The NIS is designed to approximate a $20 \%$ sample of US community hospitals, defined by the American Hospital Association as "all nonfederal, short-term, general, and other specialty hospitals, excluding hospital units of institutions." The NIS hospital sample is drawn from states participating in HCUP. For 2009, these 44 states comprise more than $95 \%$ of the US population. This universe of US community hospitals is divided into strata by 5 hospital characteristics: ownership/control, number of beds, teaching status, urban/rural location, and US region. The NIS is a stratified probability sample of hospitals; sampling probabilities are proportional to the number of US community hospitals in each stratum. The 2009 NIS includes all discharge data from 1,050 hospitals that were selected for the sample, a total of 7,810,762 unweighted discharges. Sample weights are provided to produce national estimates.

The study population is restricted to adult patients aged 18 or older admitted for diagnoses other than pregnancy and maternity. The expected payers are defined hierarchically by using primary and secondary expected payer as Medicare, then Medicaid, privately insured, and uninsured. Some "other" categories that include private insurers and public funding are not uniformly reported, so they are included in the privately insured group. The age groups are 18 to 44 years, 45 to 64 years, and 65 or older. Race and ethnicity are defined as white, black, Hispanic, Asian/Pacific Islander, and Native American.

\section{Enhancement of hospital sample for race/ethnicity coding}

Some states and hospitals do not provide patient race or ethnicity on each discharge record. Therefore, an internal Agency for Healthcare Research and Quality (AHRQ)-enhanced version of the NIS was created to produce robust national estimates stratified by race and ethnicity. For hospitals that do not provide race/ethnicity, the enhanced database randomly selected additional hospitals in the same stratum without replacement or duplication, attempting to reach the 20\% target of hospitals in the stratum. This method of preparing data is used in the National Hospital Disparities Report $(5,6)$.

\section{Calculation of the number of chronic conditions}

We used the set of chronic conditions developed by the Department of Health and Human Services (HHS) Interagency Workgroup on MCC and the Office of the Assistant Secretary of Health (7). Highly related diagnoses of the same condition were grouped together using an established Clinical Classification System (CCS) (8). The CCS categories were matched to the established set of chronic conditions; an exception was made for Autism Spectrum Disorder, for which individual ICD-9-CM codes were used. Steps were taken to avoid overcounting the number of 15 conditions reported on a single discharge summary. No one CCS category was counted more than once. In addition, we grouped clusters of CCS categories for highly related conditions (eg, 2 CCS categories for diabetes are clustered together). No cluster is counted more than once. The criteria used to identify the 15 chronic conditions are provided in an Appendix. Chronic conditions on each discharge record were counted and grouped into 3 categories (o-1, 2-3, and 4 or more). Multiple chronic conditions (MCC) are defined as 2 or more chronic conditions.

\section{Cost and mortality}

Cost is an estimate of resources used in production of service and includes direct hospital costs without physician fees. All-payer, inpatient cost-to-charge ratios are constructed from the hospital's accounting data for 2009 as reported to the Centers for Medicare and Medicaid Services (CMS). For 10\% to 15\% of hospitals, cost-to-charge ratios are estimated by imputation within state by hospital characteristics (9). Mortality rates represent only deaths in the hospital.

\section{Results}

We calculated a national estimate of the overall distribution of multiple chronic conditions for adult discharges by age group, expected primary payer, and sex (Table 1). Each column shows several use and cost items for the number of chronic conditions (o-1, 2-3, 4 or more), including the mortality rate, mean length of stay, and cost. Roughly one-third of discharges were in the highest MCC grouping ( 4 or more). The mortality rate was higher for adults discharged with 4 or more MCC compared with that of adults with $0-1$ chronic conditions (3.1\% vs 1.9\%); there was a longer length of stay and a $9 \%$ higher cost per discharge.

Medicare covers $53.7 \%$ of all the discharges and has a higher share of the discharges with 4 or more MCC (74.8\%). Fewer than half (46\%) of Medicare discharges had 4 or more chronic conditions. For privately insured patients, only $16.7 \%$ had 4 or more chronic conditions. Men and women both had about one-third of discharges with 4 or more 
chronic conditions. The differences by age are striking. Only $6.5 \%$ of discharges aged 18 to 44 years had 4 or more chronic conditions, whereas adults aged 65 or older had rates similar to those of the entire Medicare population (47.5\% with 4 or more chronic conditions). We found small differences in the distribution of chronic conditions by racial/ethnic groups. The proportion of adults discharged with 4 or more chronic conditions was lowest for Hispanics (26.3\%) and highest for whites (33.7\%). Asian/Pacific Islanders had the highest mortality regardless of number of chronic conditions, and the highest costs per case $(\$ 14,000)$ compared with $\$ 11,000$ for all groups combined.

The distribution of discharges for different payer categories was nested within age and sex (Table 2). In this context, substantial differences can be seen in the Medicaid-covered population by age group. Younger adults covered by Medicaid have a relatively low percentage of adults with 4 or more MCC ( $9 \%$ for men, $7.7 \%$ for women). The percentage for each sex rises to about $32 \%$ for adults aged 45 through 64 and then to $42 \%$ for adults aged 65 or older. Uninsured adults and adults with private payers had a lower percentage of discharges with 4 or more MCC across all age groups and each sex.

We analyzed differences in distribution and outcome by racial/ethnic groups nested within age groups and sex (Table 3). In younger age groups, a higher proportion of black men discharged have 4 or more MCC than do any other racial/ethnic group. This same was true of black women aged 18 through 44. Differences among adults by race and ethnicity are hidden when ages are combined.

We identified the most common pairs of conditions nested within age and sex for adults discharged with 2 or more conditions (Table 4). For example, an estimated 1,044,459 adult men aged 18 through 44 have 2 or more chronic conditions on their discharge abstract. Of those, approximately $24 \%$ have the dyad of depression and substance abuse. Clearly, hypertension is found in most of these combinations. After age 44, two-way combinations of coronary artery disease, diabetes, hyperlipidemia, and hypertension are the most prominent dyads.

Triads of chronic conditions reveal a few additional conditions beyond those demonstrated within the most common dyads (Table 5). We determined the 5 most common triads of the 15 chronic condition groups, nested within age and sex. Chronic kidney disease and then, after age 44, cardiac arrhythmia make their way into the most frequent triads.

\section{Summary}

An estimated 20 million adult discharges from community hospitals in the United States have 2 or more chronic conditions noted on their hospital record. These discharges include nearly $66 \%$ of all adult discharges from US hospitals. More than 9 million adult discharges (almost a third of all discharges) are estimated to have 4 or more chronic conditions. These data demonstrate compelling findings to support the fourth goal of the HHS Multiple Chronic Conditions Strategic Framework by providing the detailed distribution of multiple chronic conditions among adult discharges from community hospitals. The data suggest that payer group and racial/ethnic groups are associated with the number of chronic conditions listed in a hospital discharge summary, as are cost per stay, mortality rate, stays per year, and cost per year. Although the causal underpinnings of the associations are not explored here, the differences in cost, length of stay, and mortality for patients with 4 or more chronic conditions are substantial compared with those for adults with 1 chronic condition or none. Given that hospital costs remain the largest component of health care spending, the concentration of use and cost among patients with MCC demonstrates the need for a sustained effort to identify and treat MCC. Many factors could influence the incidence and management of chronic illness that are confounded with demographic and payer categories or operate differently in different groups.

Although the HCUP NIS is a singular source for national estimates of all-payer hospital-based use, outcomes, and cost, this database has strengths and limitations for examining MCCs. For example, while the HCUP NIS includes use and cost for private, public, and uninsured patients, it is limited to the experience of hospitalized adults. The data do not include outpatient costs or physician costs associated with MCC treatment. In addition, the data are not at the patient level but at the discharge level, so that use of frequently readmitted patients to the hospital is included in the database.

An increased number of these 15 chronic conditions for any hospitalized adult are associated with higher cost per stay and higher mortality. The well-known association between increasing age and number of chronic conditions is demonstrated in our study as well. However, nesting payer or racial/ethnic groups within age and sex highlights important associations by age and sex. These findings may help public health agencies and private health plans to identify subpopulations that will have higher costs and poorer outcomes. This information might be used in designing and targeting new services, patient education, or financial incentives to support effective management of complex chronic illness. Once implemented, these data can also help evaluate the impact of new clinical or delivery system strategies on hospital use, outcomes, or cost. 


\section{Acknowledgments}

The authors were supported wholly by the Agency for Healthcare Research and Quality during the conduct of this work. The views are those of the authors. No official endorsement by any agency of the federal or state governments is intended or should be inferred.

We are grateful to Valeriy Bakaushin and Ed Hock of Social and Scientific Systems, Inc, and to Clare Sun of ThomsonReuters, Inc for programming, and to the following state partners supplying data: Arizona Department of Health Services; Arkansas Department of Health; California Office of Statewide Health Planning and Development; Colorado Hospital Association; Connecticut Hospital Association; Florida Agency for Health Care Administration; Georgia Hospital Association; Hawaii Health Information Corporation; Illinois Department of Public Health; Indiana Hospital Association; Iowa Hospital Association; Kansas Hospital Association; Kentucky Cabinet for Health and Family Services; Louisiana Department of Health and Hospitals; Maine Health Data Organization; Maryland Health Services Cost Review Commission; Massachusetts Division of Health Care Finance and Policy; Michigan Health and Hospital Association; Minnesota Hospital Association; Missouri Hospital Industry Data Institute; Nebraska Hospital Association; Nevada Department of Health and Human Services; New Hampshire Department of Health and Human Services; New Jersey Department of Health and Senior Services; New Mexico Health Policy Commission; New York State Department of Health; North Carolina Department of Health and Human Services; Ohio Hospital Association; Oklahoma State Department of Health, Oregon Association of Hospitals and Health Systems; Pennsylvania Health Care Cost Containment Council; Rhode Island Department of Health; South Carolina State Budget and Control Board; South Dakota Association of Healthcare Organizations; Tennessee Hospital Association; Texas Department of State Health Services; Utah Department of Health; Vermont Association of Hospitals and Health Systems; Virginia Health Information; Washington State Department of Health; West Virginia Health Care Authority; Wisconsin Department of Health Services; Wyoming Hospital Association.

\section{Author Information}

Corresponding Author: Claudia A. Steiner, MD, MPH, Senior Research Physician, Healthcare Cost and Utilization Project, Center for Delivery, Organization and Markets, Agency for Healthcare Research and Quality, 540 Gaither Rd, Rockville, MD 20850. Telephone: 301-427-1407. E-mail: Claudia.Steiner@ahrq.hhs.gov.

Author Affiliations: Bernard Friedman, Agency for Healthcare Research and Quality, Rockville, Maryland.

\section{References}

1. Partnership for Solutions. A Project of Johns Hopkins University and The Robert Wood Johnson Foundation; 2001-2008; http://www.partnershipforsolutions.org. Accessed February 27, 2013.

2. Friedman B, Jiang J, Elixhauser A, Segal A. Hospital inpatient costs for adults with multiple chronic conditions. Med Care Res Rev 2006;63(3):327-46.

3. Friedman B, Jiang J, Elixhauser A. Costly hospital readmissions and complex chronic illness. Inquiry 20082009;45(4):408-21.

4. HCUP NIS Database Documentation. Healthcare Cost and Use Project (HCUP). June 2012. Agency for Healthcare Research and Quality, Rockville, Maryland. www.hcup-us.ahrq.gov/db/nation/nis/nisdbdocumentation.jsp. Accessed February 27, 2013.

5. U.S. Department of Health and Human Services. 2010 National Healthcare Disparities Report, AHRQ Publication No. 11-0004. http://hcfgkc.org/sites/default/files/documents/2010-AHRQ-National-Healthcare-DisparitiesReport.pdf. Accessed March 7, 2013.

6. Coffey R, Barrett M, Houchens R, Moy E, Ho K, Andrews R, et al. Methods applying AHRQ quality indicators to Healthcare Cost and Use Project (HCUP) data for the ninth (2011) National Healthcare Quality Report (NHQR) and National Healthcare Disparities Report (NHDR). 2011. HCUP Methods Series Report \# 2011-06. December 9, 2011. US Agency for Healthcare Research and Quality. http://www.hcupus.ahrq.gov/reports/methods/2011_06.pdf. Accessed February 27, 2013.

7. Goodman RA, Posner SF, Huang ES, Parekh AK, Koh HK. Defining and measuring chronic conditions: imperatives for research, policy, program and practice. Prev Chronic Dis 2013;10: 120239.

8. HCUP CCS. Healthcare Cost and Use Project (HCUP). August 2012. Agency for Healthcare Research and Quality, Rockville (MD). www.hcup-us.ahrq.gov/toolssoftware/ccs/ccs.jsp. Accessed February 27, 2013.

9. Cost-to-Charge Ratio Files. Healthcare Cost and Use Project (HCUP). September 2012. Agency for Healthcare Research and Quality, Rockville, MD. www.hcup-us.ahrq.gov/db/state/costtocharge.jsp. Accessed February 27, 2013. 
Preventing Chronic Disease I Hospital Utilization, Costs, and Mortality for Adults With ... Page 5 of 20

\section{Tables}

Table 1. Adult Hospital Discharges, by Number of Chronic Conditions Across Payer, Sex, Age, Race/Ethnicity, Nationwide Inpatient Sample, 2009

\begin{tabular}{|c|c|c|c|c|}
\hline \multirow[b]{2}{*}{ Discharge Characteristic } & \multicolumn{4}{|c|}{ No. of Chronic Conditions } \\
\hline & 0 or 1 & 2 or 3 & $\geq 4$ & All \\
\hline \multicolumn{5}{|c|}{ All Adult Discharges } \\
\hline Discharges, n (\%)a & $8,167,314(28.81)$ & $10,929,300(38.56)$ & $9,252,415(32.64)$ & $28,349,029(100.00)$ \\
\hline Mortality rate & 0.02 & 0.03 & 0.03 & 0.03 \\
\hline Mean length of stay, $d$ & 4.46 & 5.21 & 5.42 & 5.06 \\
\hline Mean charge, $\$$ & $35,385.98$ & $37,602.67$ & $38,672.55$ & $37,311.28$ \\
\hline Mean cost, \$ & $10,544.91$ & $11,180.93$ & $11,480.79$ & $11,095.01$ \\
\hline \multicolumn{5}{|c|}{ Payer } \\
\hline \multicolumn{5}{|l|}{ Medicare } \\
\hline n (\%) & $2,204,737(14.54)$ & $6,033,738(39.80)$ & $6,922,039(45.66)$ & $15,160,515(100.00)$ \\
\hline Mortality rate & 0.04 & 0.03 & 0.03 & 0.03 \\
\hline Mean length of stay, $d$ & 5.62 & 5.50 & 5.51 & 5.52 \\
\hline Mean charge, \$ & 39653.15 & 38123.91 & 37786.48 & 38193.47 \\
\hline Mean cost, $\$$ & $11,922.59$ & $11,394.49$ & $11,284.88$ & $11,421.66$ \\
\hline \multicolumn{5}{|l|}{ Medicaid } \\
\hline $\mathrm{n}(\%)$ & $1,181,760(37.01)$ & $1,280,715(40.11)$ & $730,609(22.88)$ & $3,193,083(100.00)$ \\
\hline Mortality rate & 0.02 & 0.02 & 0.02 & 0.02 \\
\hline Mean length of stay, $d$ & 5.26 & 5.95 & 5.89 & 5.68 \\
\hline Mean charge, $\$$ & $37,242.88$ & $36,467.74$ & $39,491.62$ & $37,445.82$ \\
\hline Mean cost, $\$$ & $10,634.06$ & $10,398.02$ & $11,244.41$ & $10,678.85$ \\
\hline \multicolumn{5}{|l|}{ Private } \\
\hline n (\%) & $3,875,344(47.10)$ & $2,977,623(36.19)$ & $1,374,750(16.71)$ & $8,227,717(100.00)$ \\
\hline Mortality rate & 0.01 & 0.02 & 0.02 & 0.02 \\
\hline Mean length of stay, $d$ & 3.72 & 4.45 & 4.82 & 4.17 \\
\hline Mean charge, $\$$ & $34,257.60$ & $38,879.88$ & $43,095.20$ & $37,397.61$ \\
\hline Mean cost, \$ & $10,277.17$ & $11,626.10$ & $12,693.26$ & $11,166.47$ \\
\hline \multicolumn{5}{|l|}{ Self-pay } \\
\hline n (\%) & $905,473(51.22)$ & $637,224(36.05)$ & $225,017(12.73)$ & $1,767,713(100.00)$ \\
\hline Mortality rate & 0.01 & 0.02 & 0.02 & 0.01 \\
\hline Mean length of stay, d & 3.75 & 4.49 & 4.97 & 4.17 \\
\hline Mean charge, $\$$ & 27,386 & $29,088.97$ & $36,492.51$ & $29,160.09$ \\
\hline Mean cost, $\$$ & $8,218.75$ & $8,691.24$ & $10,945.66$ & $8,736.48$ \\
\hline \multicolumn{5}{|c|}{ Sex } \\
\hline \multicolumn{5}{|l|}{ Men } \\
\hline n (\%) & $3,582,447(27.41)$ & $5,072,042(38.80)$ & $4,417,230(33.79)$ & $13,071,719(100.00)$ \\
\hline
\end{tabular}


Preventing Chronic Disease I Hospital Utilization, Costs, and Mortality for Adults With ... Page 6 of 20

\begin{tabular}{|c|c|c|c|c|}
\hline \multirow[b]{2}{*}{ Discharge Characteristic } & \multicolumn{4}{|c|}{ No. of Chronic Conditions } \\
\hline & 0 or 1 & 2 or 3 & $\geq 4$ & All \\
\hline Mortality rate & 0.02 & 0.02 & 0.03 & 0.02 \\
\hline Mean length of stay, $d$ & 4.19 & 5.13 & 5.45 & 4.95 \\
\hline Mean charge, $\$$ & $38,860.38$ & $40,070.49$ & $41,303.33$ & $40,153.68$ \\
\hline Mean cost, $\$$ & $11,524.23$ & $11,873.24$ & $12,207.98$ & $11,890.22$ \\
\hline \multicolumn{5}{|l|}{ Women } \\
\hline n (\%) & $4,569,669(29.89)$ & $5,871,812(38.41)$ & $4,844,323(31.69)$ & $15,285,805(100.00)$ \\
\hline Mortality rate & 0.02 & 0.02 & 0.03 & 0.02 \\
\hline Mean length of stay, d & 4.19 & 5.13 & 5.45 & 4.95 \\
\hline Mean charge, $\$$ & $32,646.41$ & $35,449.35$ & $36,266.74$ & $34,868.34$ \\
\hline Mean cost, $\$$ & $9,790.54$ & $10,583.73$ & $10,820.74$ & $10,421.11$ \\
\hline \multicolumn{5}{|c|}{ Age } \\
\hline \multicolumn{5}{|l|}{$18-44 y$} \\
\hline n (\%) & $3,419,009(63.36)$ & $1,624,350(30.10)$ & $352,970.50(6.54)$ & $5,396,330(100.00)$ \\
\hline Mortality rate & 0.01 & 0.01 & 0.01 & 0.01 \\
\hline Mean length of stay, $d$ & 3.91 & 5.03 & 5.20 & 4.33 \\
\hline Mean charge, $\$$ & $30,804.92$ & $30,364.65$ & $34,111.91$ & $30,910.92$ \\
\hline Mean cost, $\$$ & $9,142.41$ & $9,025.33$ & $10,080.57$ & $9,168.51$ \\
\hline \multicolumn{5}{|l|}{$45-64 y$} \\
\hline n (\%) & $2,992,037(31.60)$ & $3,987,333(42.11)$ & $2,488,444(26.28)$ & $9,467,814(100.00)$ \\
\hline Mortality rate & 0.02 & 0.02 & 0.02 & 0.02 \\
\hline Mean length of stay, $d$ & 4.50 & 5.05 & 5.26 & 4.93 \\
\hline Mean charge, $\$$ & $37,773.66$ & $39,377.85$ & $40,983.65$ & $39,292.56$ \\
\hline Mean cost, $\$$ & $11,299.41$ & $11,710.14$ & $12,107.17$ & $11,684.60$ \\
\hline \multicolumn{5}{|l|}{$\geq 65 y$} \\
\hline Discharges, n (\%) & $1,774,032(13.11)$ & $5,334,250(39.43)$ & $6,420,323(47.46)$ & $13,528,605(100.00)$ \\
\hline Mortality rate & 0.05 & 0.04 & 0.04 & 0.04 \\
\hline Mean length of stay, $d$ & 5.44 & 5.38 & 5.50 & 5.44 \\
\hline Mean charge, $\$$ & $40,068.53$ & $38,467.40$ & $38,022.14$ & $38,467.45$ \\
\hline Mean cost, \$ & $11,974.46$ & $11,444.67$ & $11,317.35$ & $11,454.16$ \\
\hline \multicolumn{5}{|c|}{ Race/Ethnicity } \\
\hline \multicolumn{5}{|l|}{ White } \\
\hline n (\%) & $5,666,925(27.62)$ & $7,942,189(38.70)$ & $6,911,652(33.68)$ & $20,520,766(100.00)$ \\
\hline Mortality rate & 0.02 & 0.03 & 0.03 & 0.03 \\
\hline Mean length of stay, $d$ & 4.37 & 5.06 & 5.30 & 4.95 \\
\hline Mean charge, $\$$ & $34,050.26$ & $35,737.80$ & $36,542.40$ & $35,541.02$ \\
\hline Mean cost, \$ & $10,576.29$ & $11,075.09$ & $11,266.23$ & $11,001.25$ \\
\hline \multicolumn{5}{|l|}{ Black } \\
\hline n (\%) & $1,045,058(27.01)$ & $1,546,737(39.98)$ & $1,277,201(33.01)$ & $3,868,996(100.00)$ \\
\hline
\end{tabular}


Preventing Chronic Disease I Hospital Utilization, Costs, and Mortality for Adults With ... Page 7 of 20

\begin{tabular}{|c|c|c|c|c|}
\hline \multirow[b]{2}{*}{ Discharge Characteristic } & \multicolumn{4}{|c|}{ No. of Chronic Conditions } \\
\hline & 0 or 1 & 2 or 3 & $\geq 4$ & All \\
\hline Mortality rate & 0.02 & 0.02 & 0.02 & 0.02 \\
\hline Mean length of stay, $d$ & 4.99 & 5.74 & 5.81 & 5.56 \\
\hline Mean charge, $\$$ & $35,929.6$ & $38,679.35$ & $39,139.03$ & $38,087.77$ \\
\hline Mean cost, $\$$ & $10,114.23$ & $10,877.67$ & $11,276.55$ & $10,802.8$ \\
\hline \multicolumn{5}{|l|}{ Hispanic } \\
\hline n (\%) & $890,011(37.49)$ & $859,422(36.20)$ & $624,850(26.32)$ & $2,374,283(100.00$ \\
\hline Mortality rate & 0.02 & 0.02 & 0.03 & 0.02 \\
\hline Mean length of stay, d & 4.37 & 5.37 & 5.74 & 5.10 \\
\hline Mean charge, $\$$ & $39,520.76$ & $45,699.29$ & $51,147.17$ & $44,807.2 \varepsilon$ \\
\hline Mean cost, $\$$ & $10,241.91$ & $11,319.79$ & $12,467.65$ & $11,215.95$ \\
\hline \multicolumn{5}{|l|}{ Asian/Pacific Islander } \\
\hline n (\%) & $162,048(31.36)$ & $196,067(37.94)$ & $158,688(30.71)$ & $516,804(100.00)$ \\
\hline Mortality rate & 0.03 & 0.04 & 0.04 & 0.03 \\
\hline Mean length of stay, $d$ & 4.80 & 5.68 & 5.92 & $5.4 \varepsilon$ \\
\hline Mean charge, $\$$ & $47,747.97$ & $57,105.99$ & $64,097.02$ & $56,257.44$ \\
\hline Mean cost, \$ & $12,455.85$ & $14,280.97$ & $15,380.64$ & $14,035.66$ \\
\hline \multicolumn{5}{|l|}{ Native American } \\
\hline n (\%) & $61,241.47(31.16)$ & $76,597.34(38.97)$ & $58,721.93(29.87)$ & $196,560.70(100.00$ \\
\hline Mortality rate & 0.02 & 0.02 & 0.03 & 0.02 \\
\hline Mean length of stay, $d$ & 4.35 & 5.05 & 5.03 & 4.83 \\
\hline Mean charge, \$ & $30,114.33$ & $32,991.89$ & $37,077.33$ & $33,314.41$ \\
\hline Mean cost, $\$$ & $10,368.74$ & $11,160.54$ & $11,499.38$ & $11,014.92$ \\
\hline
\end{tabular}

a The number of all discharges within the columns of each detailed breakdown section of the table may not add precisely to all discharges in the first line of the table because of missing data on national estimates within each section. For more information on sampling variation and missing data for particular variables, consult www.hcupnet.ahrq.gov.

Table 2. Adults Discharged From US Hospitals by Payer Within Age and Sex, Nationwide Inpatient Sample, 2009

\begin{tabular}{|c|c|c|c|c|}
\hline \multirow[b]{2}{*}{ Discharge Characteristic } & \multicolumn{4}{|c|}{ No. of Chronic Conditions } \\
\hline & 0 to 1 & 2 to 3 & $\geq 4$ & All \\
\hline \multicolumn{5}{|c|}{ Men aged 18-44 y } \\
\hline \multicolumn{5}{|l|}{ Medicare } \\
\hline n (\%) & $108,888(34.78)$ & $145,761(46.56)$ & $58,433(18.66)$ & $313,082(100.00)$ \\
\hline Mortality rate & 0.01 & 0.01 & 0.01 & 0.01 \\
\hline Mean length of stay, $d$ & 6.31 & 6.30 & 5.79 & 6.21 \\
\hline Mean charge, $\$$ & $36,282.56$ & $33,043.79$ & $34,346.26$ & $34,413.36$ \\
\hline Mean cost, $\$$ & $10,765.5$ & $9,784.298$ & $10,156.62$ & $10,195.06$ \\
\hline
\end{tabular}


Preventing Chronic Disease I Hospital Utilization, Costs, and Mortality for Adults With ... Page 8 of 20

\begin{tabular}{|c|c|c|c|c|}
\hline \multirow[b]{2}{*}{ Discharge Characteristic } & \multicolumn{4}{|c|}{ No. of Chronic Conditions } \\
\hline & 0 to 1 & 2 to 3 & $\geq 4$ & All \\
\hline n (\%) & $312,401(51.81)$ & $236,287(39.18)$ & $54,321(9.01)$ & $603,009(100.00)$ \\
\hline Mortality rate & 0.01 & 0.01 & 0.01 & 0.01 \\
\hline Mean length of stay, $d$ & 3.78 & 4.49 & 4.52 & 4.01 \\
\hline Mean charge, $\$$ & $34,158.33$ & $32,689.01$ & $38,973.72$ & $33,976.47$ \\
\hline Mean cost, $\$$ & $10,066.80$ & $9,690.28$ & $11,398.59$ & $10,024.63$ \\
\hline \multicolumn{5}{|l|}{ Private } \\
\hline n (\%) & $758,767(68.21)$ & $303,074(27.25)$ & $50,517(4.54)$ & $1,112,358(100.00)$ \\
\hline Mortality rate & 0.01 & 0.01 & 0.01 & 0.01 \\
\hline Mean length of stay, d & 3.78 & 4.49 & 4.52 & 4.01 \\
\hline Mean charge, \$ & $34,158.33$ & $32,689.01$ & $38,973.72$ & $33,976.47$ \\
\hline Mean cost, \$ & 10066.80 & $9,690.28$ & $11,398.59$ & $10,024.63$ \\
\hline \multicolumn{5}{|l|}{ Self-pay } \\
\hline n (\%) & $351,196(64.58)$ & $166,152(30.55)$ & $26,455(4.86)$ & $543,804(100.00)$ \\
\hline Mortality rate & 0.01 & 0.01 & 0.01 & 0.01 \\
\hline Mean length of stay, $d$ & 3.65 & 4.37 & 4.39 & 3.91 \\
\hline Mean charge, $\$$ & $25,756.81$ & $23,822.86$ & $30,858.46$ & $25,414.02$ \\
\hline Mean cost, $\$$ & $7,793.37$ & $7,160.63$ & $9,027.34$ & $7,660.02$ \\
\hline \multicolumn{5}{|c|}{ Men aged 45-64 y } \\
\hline \multicolumn{5}{|l|}{ Medicare } \\
\hline n (\%) & $203,301(17.84)$ & $467,741(41.04)$ & $468,674(41.12)$ & $1,139,716(100.00)$ \\
\hline Mortality rate & 0.03 & 0.02 & 0.02 & 0.02 \\
\hline Mean length of stay, d & 6.55 & 6.09 & 5.56 & 5.95 \\
\hline Mean charge, $\$$ & $44,284.56$ & $40,537.61$ & $39,541.04$ & $40,796.60$ \\
\hline Mean cost, $\$$ & $13,245.30$ & $11,960.11$ & $11,666.46$ & $12,068.74$ \\
\hline \multicolumn{5}{|l|}{ Medicaid } \\
\hline n (\%) & $182,430(23.77)$ & $343,745(44.80)$ & $241,169(31.43)$ & $767,344(100.00)$ \\
\hline Mortality rate & 0.03 & 0.02 & 0.02 & 0.02 \\
\hline Mean length of stay, d & 6.75 & 6.41 & 5.95 & 6.35 \\
\hline Mean charge, $\$$ & $45,922.70$ & $40,771.91$ & $40,881.27$ & $42,030.43$ \\
\hline Mean cost, $\$$ & $13,085.48$ & $11,751.80$ & $11,711.18$ & $12,056.01$ \\
\hline \multicolumn{5}{|l|}{ Private } \\
\hline $\mathrm{n}(\%)$ & $813,767(33.87)$ & $1,051,276(43.76)$ & $537,256(22.36)$ & $2,402,299(100.00)$ \\
\hline Mortality rate & 0.02 & 0.02 & 0.01 & 0.02 \\
\hline Mean length of stay, d & 4.24 & 4.35 & 4.64 & 4.38 \\
\hline Mean charge, $\$$ & $40,495.69$ & $42,715.50$ & $46,321.88$ & $42,767.70$ \\
\hline Mean cost, \$ & $12,150.26$ & $12,752.72$ & $13,698.33$ & $12,759.49$ \\
\hline \multicolumn{5}{|l|}{ Self-pay } \\
\hline n (\%) & $166,307(35.49)$ & $205,565(43.87)$ & $96,733(20.64)$ & $468,605(100.00)$ \\
\hline
\end{tabular}


Preventing Chronic Disease I Hospital Utilization, Costs, and Mortality for Adults With ... Page 9 of 20

\begin{tabular}{|c|c|c|c|c|}
\hline \multirow[b]{2}{*}{ Discharge Characteristic } & \multicolumn{4}{|c|}{ No. of Chronic Conditions } \\
\hline & 0 to 1 & 2 to 3 & $\geq 4$ & All \\
\hline Mortality rate & 0.02 & 0.02 & 0.02 & 0.02 \\
\hline Mean length of stay, $d$ & 4.56 & 4.78 & 5.01 & 4.75 \\
\hline Mean charge, \$ & $31,775.72$ & $33,344.77$ & $39,030.38$ & $33,961.61$ \\
\hline Mean cost, $\$$ & $9,579.73$ & $9,992.05$ & $11,507.92$ & $10,158.64$ \\
\hline \multicolumn{5}{|c|}{ Men aged $\geq 65 y$} \\
\hline \multicolumn{5}{|l|}{ Medicare } \\
\hline n (\%) & $665,135(12.62)$ & $2,014,678(38.24)$ & $2,588,693(49.14)$ & $5,268,507(100.00)$ \\
\hline Mortality rate & 0.05 & 0.04 & 0.04 & 0.04 \\
\hline Mean length of stay, d & 5.81 & 5.48 & 5.43 & 5.50 \\
\hline Mean charge, $\$$ & $43,426.57$ & $41,082.58$ & $39,831.74$ & $40,765.79$ \\
\hline Mean cost, $\$$ & $13,121.63$ & $12,303.19$ & $11,882.03$ & $12,200.22$ \\
\hline \multicolumn{5}{|l|}{ Medicaid } \\
\hline n (\%) & $14,603(16.51)$ & $37,212(42.07)$ & $36,630(41.42)$ & $88,445(100.00)$ \\
\hline Mortality rate & 0.06 & 0.05 & 0.04 & 0.05 \\
\hline Mean length of stay, $d$ & 7.20 & 7.07 & 7.21 & 7.15 \\
\hline Mean charge, $\$$ & $52,644.60$ & $48,780.02$ & $48,914.96$ & $49,474.11$ \\
\hline Mean cost, $\$$ & $14,215.67$ & $13,230.12$ & $13,480.00$ & $13,496.22$ \\
\hline \multicolumn{5}{|l|}{ Private } \\
\hline Discharges, n (\%) & $76,922(16.40)$ & $189,141(40.31)$ & $203,115(43.29)$ & $469,177(100.00)$ \\
\hline Mortality rate & 0.08 & 0.06 & 0.05 & 0.06 \\
\hline Mean length of stay, $d$ & 5.30 & 5.13 & 5.21 & 5.19 \\
\hline Mean charge, $\$$ & $45,328.43$ & $44,083.07$ & $43,782.92$ & $44,158.40$ \\
\hline Mean cost, $\$$ & $13,026.96$ & $12,629.63$ & $12,440.45$ & $12,613.35$ \\
\hline \multicolumn{5}{|l|}{ Self-pay } \\
\hline n (\%) & $7,836(21.69)$ & $14,798(40.95)$ & $13,501(37.36)$ & $36,136(100.00)$ \\
\hline Mortality rate & 0.108 & 0.06 & 0.06 & 0.07 \\
\hline Mean length of stay, $d$ & 5.58 & 5.54 & 6.17 & 5.79 \\
\hline Mean charge, \$ & $36,910.76$ & $39,488.91$ & $39,595.57$ & $38,970.75$ \\
\hline Mean cost, $\$$ & $11,220.25$ & $12,522.91$ & $12,651.25$ & $12,288.72$ \\
\hline \multicolumn{5}{|c|}{ Women aged 18-44 y } \\
\hline \multicolumn{5}{|l|}{ Medicare } \\
\hline $\mathrm{n}(\%)$ & $119,666(38.58)$ & $140,524(45.31)$ & $49,967(16.11)$ & $310,157(100.00)$ \\
\hline Mortality rate & 0.01 & 0.01 & 0.01 & 0.01 \\
\hline Mean length of stay, d & 5.49 & 5.90 & 5.54 & 5.69 \\
\hline Mean charge, $\$$ & $32,332.45$ & $33,295.78$ & $33,724.04$ & $32,992.90$ \\
\hline Mean cost, $\$$ & $9,748.48$ & $9,783.22$ & $9,955.79$ & $9,797.59$ \\
\hline \multicolumn{5}{|l|}{ Medicaid } \\
\hline n (\%) & $4,492,580(58.93)$ & $278,647(33.34)$ & $64,634(7.73)$ & $835,860(100.00)$ \\
\hline
\end{tabular}


Preventing Chronic Disease I Hospital Utilization, Costs, and Mortality for Adults With... Page 10 of 20

\begin{tabular}{|c|c|c|c|c|}
\hline \multirow[b]{2}{*}{ Discharge Characteristic } & \multicolumn{4}{|c|}{ No. of Chronic Conditions } \\
\hline & 0 to 1 & 2 to 3 & $\geq 4$ & All \\
\hline Mortality rate & 0.01 & 0.01 & 0.01 & 0.01 \\
\hline Mean length of stay, $d$ & 4.24 & 5.31 & 5.32 & 4.68 \\
\hline Mean charge, $\$$ & $28,398.89$ & $27,609.89$ & $30,565.52$ & $28,303.17$ \\
\hline Mean cost, $\$$ & $8,376.36$ & $8,193.98$ & $9,117.12$ & $8,372.78$ \\
\hline \multicolumn{5}{|l|}{ Private } \\
\hline n (\%) & $1,048,248(75.45)$ & $303,657(21.86)$ & $37,472(2.70)$ & $1,389,376(100.00)$ \\
\hline Mortality rate & 0.00 & 0.01 & 0.01 & 0.00 \\
\hline Mean length of stay, $d$ & 3.21 & 4.24 & 4.47 & 3.47 \\
\hline Mean charge, $\$$ & $27,901.65$ & $29,707.63$ & $34,695.92$ & $28,478.33$ \\
\hline Mean cost, $\$$ & $8,312.62$ & $8,834.00$ & $10,243.32$ & $8,478.28$ \\
\hline \multicolumn{5}{|l|}{ Self-pay } \\
\hline n (\%) & $248,365(69.35)$ & $96,378(26.91)$ & $13,406(2.70)$ & $358,149(100.00)$ \\
\hline Mortality rate & 0.00 & 0.01 & 0.01 & 0.01 \\
\hline Mean length of stay, $d$ & 3.27 & 4.10 & 4.40 & 3.54 \\
\hline Mean charge, $\$$ & $22,693.59$ & $22,365.63$ & $29,013.77$ & $22,842.02$ \\
\hline Mean cost, $\$$ & $6,926.04$ & $6,738.00$ & $8,623.15$ & $6,938.96$ \\
\hline \multicolumn{5}{|c|}{ Women aged 45-64 y } \\
\hline \multicolumn{5}{|l|}{ Medicare } \\
\hline $\mathrm{n}(\%)$ & $224,202(20.00)$ & $474,563(42.34)$ & $422,019(37.65)$ & $1,120,783(100.00)$ \\
\hline Mortality rate & 0.02 & 0.02 & 0.02 & 0.02 \\
\hline Mean length of stay, $d$ & 6.00 & 5.84 & 5.54 & 5.76 \\
\hline Mean charge, $\$$ & $39,567.05$ & $37,455.52$ & $37,136.58$ & $37,758.12$ \\
\hline Mean cost, \$ & $11,987.25$ & $11,229.84$ & $11,025.40$ & $11,304.51$ \\
\hline \multicolumn{5}{|l|}{ Medicaid } \\
\hline n (\%) & $210,734(24.40)$ & $373,959(43.30)$ & $278,978(32.30)$ & $863,671(100.00)$ \\
\hline Mortality rate & 0.02 & 0.02 & 0.01 & 0.02 \\
\hline Mean length of stay, $d$ & 5.75 & 5.85 & 5.70 & 5.78 \\
\hline Mean charge, $\$$ & $39,336.88$ & $37,011.21$ & $36,886.57$ & $37,538.15$ \\
\hline Mean cost, $\$$ & $11,395.97$ & $10,680.02$ & $10,791.38$ & $10,890.61$ \\
\hline \multicolumn{5}{|l|}{ Private } \\
\hline n (\%) & $1,080,447(44.02)$ & $988,812(40.29)$ & 385,165 (15.69) & $2,454,423(100.00)$ \\
\hline Mortality rate & 0.01 & 0.01 & 0.01 & 0.01 \\
\hline Mean length of stay, d & 3.73 & 4.31 & 4.63 & 4.11 \\
\hline Mean charge, $\$$ & $34,117.03$ & $37,245.12$ & $39,641.79$ & $36,240.15$ \\
\hline Mean cost, $\$$ & $10,300.35$ & $11,264.37$ & $11,941.66$ & $10,945.07$ \\
\hline \multicolumn{5}{|l|}{ Self-pay } \\
\hline n (\%) & $143,013(39.43)$ & $154,460(42.59)$ & $65,219(17.98)$ & $362,692(100.00)$ \\
\hline Mortality rate & 0.02 & 0.01 & 0.02 & 0.02 \\
\hline
\end{tabular}


Preventing Chronic Disease I Hospital Utilization, Costs, and Mortality for Adults With... Page 11 of 20

\begin{tabular}{|c|c|c|c|c|}
\hline \multirow[b]{2}{*}{ Discharge Characteristic } & \multicolumn{4}{|c|}{ No. of Chronic Conditions } \\
\hline & 0 to 1 & 2 to 3 & $\geq 4$ & All \\
\hline Mean length of stay, $d$ & 3.97 & 4.49 & 4.73 & 4.33 \\
\hline Mean charge, $\$$ & $27,568.26$ & $29,511.21$ & $33,909.31$ & $29,536.17$ \\
\hline Mean cost, \$ & $8,578.13$ & $9,032.98$ & $10,415.54$ & $9,102.30$ \\
\hline \multicolumn{5}{|c|}{ Women aged $\geq 65$} \\
\hline \multicolumn{5}{|l|}{ Medicare } \\
\hline n (\%) & $936,232(13.35)$ & $2,873,933(40.97)$ & $3,205,064(45.69)$ & $7,015,230(100.00)$ \\
\hline Mortality rate & 0.04 & 0.03 & 0.03 & 0.03 \\
\hline Mean length of stay, d & 5.45 & 5.30 & 5.46 & 5.39 \\
\hline Mean charge, $\$$ & $37,312.62$ & $34,902.96$ & $34,767.87$ & $35,163.93$ \\
\hline Mean cost, $\$$ & $11,430.65$ & $10,588.52$ & $10,490.42$ & $10,656.53$ \\
\hline \multicolumn{5}{|l|}{ Medicaid } \\
\hline n (\%) & $21,863(14.60)$ & $62,869(41.97)$ & $65,057(43.43)$ & $149,789(100.00)$ \\
\hline Mortality rate & 0.05 & 0.04 & 0.03 & 0.04 \\
\hline Mean length of stay, $d$ & 6.36 & 6.38 & 6.99 & 6.65 \\
\hline Mean charge, $\$$ & $42,907.69$ & $40,707.18$ & $42,918.38$ & $41,988.29$ \\
\hline Mean cost, $\$$ & $11,977.38$ & $11,338.54$ & $11,972.17$ & $11,706.85$ \\
\hline \multicolumn{5}{|l|}{ Private } \\
\hline n (\%) & $83,905(18.33)$ & $197,581(43.17)$ & $176,220(38.50)$ & $457,706(100.00)$ \\
\hline Mortality rate & 0.07 & 0.05 & 0.05 & 0.06 \\
\hline Mean length of stay, $d$ & 4.94 & 5.01 & 5.19 & 5.06 \\
\hline Mean charge, $\$$ & $36,988.99$ & $37,399.27$ & $37,110.41$ & $37,212.95$ \\
\hline Mean cost, \$ & $10,922.90$ & $10,808.53$ & $10,692.03$ & $10,784.93$ \\
\hline \multicolumn{5}{|l|}{ Self-pay } \\
\hline n (\%) & $9,699(23.06)$ & $18,097(43.03)$ & $14,260(33.91)$ & $42,056(100.00)$ \\
\hline Mortality rate & 0.07 & 0.06 & 0.05 & 0.06 \\
\hline Mean length of stay, $d$ & 6.40 & 5.48 & 6.20 & 5.94 \\
\hline Mean charge, $\$$ & $31,791.20$ & $32,658.91$ & $34,218.72$ & $32,988.01$ \\
\hline Mean cost, $\$$ & $10,023.66$ & $10,349.79$ & $11,124.45$ & $10,537.39$ \\
\hline
\end{tabular}

Table 3. Adults Discharged From US Hospitals, by Race/Ethnicity Within Age Groups and Sex, Nationwide Inpatient Sample, 2009

\begin{tabular}{|l|r|r|r|r|}
\hline \multirow{2}{*}{ Discharge Characteristic } & \multicolumn{5}{|c|}{ No. of Chronic Conditions } \\
\cline { 2 - 6 } & $\mathbf{0}$ to $\mathbf{1}$ & \multicolumn{2}{|c|}{$\mathbf{2}$ to 3 } & \multicolumn{1}{c|}{ All } \\
\hline White & \multicolumn{5}{|c|}{ Men aged 18-44 y } \\
\hline $\mathrm{n}(\%)$ & $917,075(60.83)$ & $492,730(32.68)$ & $97,880(6.49)$ & $1,507,685(100.00)$ \\
\hline Mortality rate & 0.01 & 0.01 & 0.01 & 0.01 \\
\hline Mean length of stay, $\mathrm{d}$ & 4.10 & 4.94 & 5.06 & 4.44 \\
\hline
\end{tabular}


Preventing Chronic Disease I Hospital Utilization, Costs, and Mortality for Adults With... Page 12 of 20

\begin{tabular}{|c|c|c|c|c|}
\hline \multirow[b]{2}{*}{ Discharge Characteristic } & \multicolumn{4}{|c|}{ No. of Chronic Conditions } \\
\hline & 0 to 1 & 2 to 3 & $\geq 4$ & All \\
\hline Mean charge, $\$$ & $32,308.69$ & $28,636.78$ & $33,143.72$ & $31,161.70$ \\
\hline Mean cost, $\$$ & $9,937.46$ & $89,10.26$ & $10,198.32$ & $9,618.37$ \\
\hline \multicolumn{5}{|l|}{ Black } \\
\hline n (\%) & $238,958(49.93)$ & $181,964(38.02)$ & $57,708(12.06)$ & $478,630(100.00)$ \\
\hline Mortality rate & 0.01 & 0.01 & 0.01 & 0.01 \\
\hline Mean length of stay, d & 5.03 & 5.65 & 5.51 & 5.33 \\
\hline Mean charge, $\$$ & $34,340.21$ & $31,673.97$ & $34,212.79$ & $33,310.18$ \\
\hline Mean cost, $\$$ & $9,709.54$ & $9,155.93$ & $10,078.22$ & $9,543.23$ \\
\hline \multicolumn{5}{|l|}{ Hispanic } \\
\hline n (\%) & $22,8901(65.46)$ & $99,352(28.41)$ & $21,411(6.12)$ & $349,664(100.00)$ \\
\hline Mortality rate & 0.01 & 0.01 & 0.01 & 0.01 \\
\hline Mean length of stay, d & 4.56 & 5.59 & 5.33 & 4.90 \\
\hline Mean charge, $\$$ & $40,675.98$ & $40,163.08$ & $43,418.28$ & $40,697.90$ \\
\hline Mean cost, $\$$ & $10,928.28$ & $10,542.99$ & $11,013.78$ & $10,824.10$ \\
\hline \multicolumn{5}{|l|}{ Asian/Pacific Islander } \\
\hline n (\%) & $26,604(65.46)$ & $11,263(27.71)$ & $2,777(6.83)$ & $40,644(100.00)$ \\
\hline Mortality rate & 0.01 & 0.02 & 0.01 & 0.01 \\
\hline Mean length of stay, $d$ & 4.94 & 5.96 & 5.49 & 5.26 \\
\hline Mean charge, $\$$ & $46,928.06$ & $51,705.31$ & $55,389.21$ & $48,806.68$ \\
\hline Mean cost, $\$$ & $12,290.35$ & $13,744.53$ & $13,736.64$ & $12,786.57$ \\
\hline \multicolumn{5}{|l|}{ Native American } \\
\hline n (\%) & $12,665(56.31)$ & $8,040(35.75)$ & $1,785(7.94)$ & $22,490(100.00)$ \\
\hline Mortality rate & 0.01 & 0.01 & 0.02 & 0.01 \\
\hline Mean length of stay, $d$ & 4.24 & 4.99 & 4.72 & 4.55 \\
\hline Mean charge, $\$$ & $29,490.33$ & $26,213.56$ & $33,706.71$ & $28,648.17$ \\
\hline Mean cost, $\$$ & $9,733.02$ & $8,618.44$ & $10,976.53$ & $9,431.61$ \\
\hline \multicolumn{5}{|c|}{ Men aged $45-64$ y } \\
\hline \multicolumn{5}{|l|}{ White } \\
\hline n (\%) & $964,565(29.61)$ & $1,398,301(42.92)$ & $895,219(27.48)$ & $3,258,085(100.00)$ \\
\hline Mortality rate & 0.02 & 0.02 & 0.02 & 0.02 \\
\hline Mean length of stay, $d$ & 4.70 & 4.90 & 5.07 & 4.89 \\
\hline Mean charge, \$ & $39,740.38$ & $40,268.24$ & $41,721.88$ & $40,511.04$ \\
\hline Mean cost, $\$$ & $12,290.35$ & $12,403.48$ & $12,768.23$ & $12,470.13$ \\
\hline \multicolumn{5}{|l|}{ Black } \\
\hline n (\%) & $156,756(20.67)$ & $339,238(44.73)$ & $262,498(34.61)$ & $758,492(100.00)$ \\
\hline Mortality rate & 0.02 & 0.02 & 0.02 & 0.02 \\
\hline Mean length of stay, $d$ & 5.74 & 5.74 & 5.62 & 5.70 \\
\hline Mean charge, \$ & $42,500.38$ & $39,497.04$ & $40,095.68$ & $40,324.30$ \\
\hline
\end{tabular}


Preventing Chronic Disease I Hospital Utilization, Costs, and Mortality for Adults With... Page 13 of 20

\begin{tabular}{|c|c|c|c|c|}
\hline \multirow[b]{2}{*}{ Discharge Characteristic } & \multicolumn{4}{|c|}{ No. of Chronic Conditions } \\
\hline & 0 to 1 & 2 to 3 & $\geq 4$ & All \\
\hline Mean cost, \$ & $11,874.92$ & $11,212.07$ & $11,508.44$ & $11,451.44$ \\
\hline \multicolumn{5}{|l|}{ Hispanic } \\
\hline n (\%) & $124,063(30.99)$ & $168,557(42.10)$ & $107,739(26.91)$ & $400,359(100.00)$ \\
\hline Mortality rate & 0.02 & 0.02 & 0.02 & 0.02 \\
\hline Mean length of stay, $d$ & 5.10 & 5.48 & 5.58 & 5.39 \\
\hline Mean charge, $\$$ & $46,103.51$ & $48,804.00$ & $52,895.88$ & $49,065.06$ \\
\hline Mean cost, $\$$ & $11,925.25$ & $12,216.00$ & $13,132.87$ & $12,372.10$ \\
\hline \multicolumn{5}{|l|}{ Asian/Pacific Islander } \\
\hline n (\%) & $23,944(30.84)$ & $32,410(41.75)$ & $21,279(27.41)$ & $77,633(100.00)$ \\
\hline Mortality rate & 0.03 & 0.03 & 0.02 & 0.03 \\
\hline Mean length of stay, $d$ & 5.32 & 5.58 & 5.84 & 5.57 \\
\hline Mean charge, $\$$ & $56,263.83$ & $62,086.23$ & $71,098.07$ & $62,681.37$ \\
\hline Mean cost, $\$$ & $14,496.26$ & $15,730.86$ & $17,496.28$ & $15,817.94$ \\
\hline \multicolumn{5}{|l|}{ Native American } \\
\hline n (\%) & $9,690(26.63)$ & $15,708(43.18)$ & $10,983(30.19)$ & $36,381(100.00)$ \\
\hline Mortality rate & 0.03 & 0.02 & 0.01 & 0.02 \\
\hline Mean length of stay, $d$ & 5.09 & 4.92 & 4.79 & 4.93 \\
\hline Mean charge, $\$$ & $36,101.52$ & $35,412.00$ & $39,829.43$ & $36,931.24$ \\
\hline Mean cost, $\$$ & $12,672.81$ & $11,841.69$ & $12,230.61$ & $12,179.48$ \\
\hline \multicolumn{5}{|c|}{ Men aged $\geq 65 y$} \\
\hline \multicolumn{5}{|l|}{ White } \\
\hline n (\%) & $594,416(12.67)$ & $1,767,324(37.66)$ & $2,331,641(49.68)$ & $4,693,381(100.00)$ \\
\hline Mortality rate & 0.05 & 0.04 & 0.04 & 0.04 \\
\hline Mean length of stay, $d$ & 5.45 & 5.32 & 5.35 & 5.35 \\
\hline Mean charge, $\$$ & $41,150.03$ & $39,783.78$ & $38,603.28$ & $39,372.19$ \\
\hline Mean cost, $\$$ & $12,690.98$ & $12,229.29$ & $11,821.99$ & $12,086.05$ \\
\hline \multicolumn{5}{|l|}{ Black } \\
\hline n (\%) & $52,675(10.77)$ & $187,401(38.31)$ & $249,085(50.92)$ & $489,161(100.00)$ \\
\hline Mortality rate & 0.07 & 0.04 & 0.04 & 0.04 \\
\hline Mean length of stay, $d$ & 7.27 & 6.45 & 6.14 & 6.38 \\
\hline Mean charge, $\$$ & $52,723.93$ & $46,092.80$ & $41,837.77$ & $44,645.21$ \\
\hline Mean cost, $\$$ & $14,513.92$ & $12,679.25$ & $12,011.12$ & $12,537.51$ \\
\hline \multicolumn{5}{|l|}{ Hispanic } \\
\hline n (\%) & $54,561(14.78)$ & $144,823(39.23)$ & $169,737(45.98)$ & $369,121(100.00)$ \\
\hline Mortality rate & 0.05 & 0.04 & 0.04 & 0.04 \\
\hline Mean length of stay, $d$ & 5.76 & 5.82 & 6.03 & 5.91 \\
\hline Mean charge, $\$$ & $52,214.78$ & $53,118.44$ & $56,508.38$ & $54,535.47$ \\
\hline Mean cost, $\$$ & $12,531.53$ & $12,715.68$ & $13,583.41$ & $13,085.42$ \\
\hline
\end{tabular}


Preventing Chronic Disease I Hospital Utilization, Costs, and Mortality for Adults With... Page 14 of 20

\begin{tabular}{|c|c|c|c|c|}
\hline \multirow[b]{2}{*}{ Discharge Characteristic } & \multicolumn{4}{|c|}{ No. of Chronic Conditions } \\
\hline & 0 to 1 & 2 to 3 & $\geq 4$ & All \\
\hline \multicolumn{5}{|l|}{ Asian/Pacific Islander } \\
\hline n (\%) & $16,563(14.09)$ & $47,631(40.53)$ & $53,322(45.37)$ & $117,517(100.00)$ \\
\hline Mortality rate & 0.06 & 0.05 & 0.05 & 0.05 \\
\hline Mean length of stay, $d$ & 6.14 & 6.13 & 6.08 & 6.11 \\
\hline Mean charge, $\$$ & $62,512.13$ & $64,955.69$ & $68,702.57$ & $66,278.41$ \\
\hline Mean cost, \$ & $15,711.12$ & $15,857.96$ & $16,261.18$ & $16,016.96$ \\
\hline \multicolumn{5}{|l|}{ Native American } \\
\hline n (\%) & $4,597(14.04)$ & $12,286(37.53)$ & $15,855(48.43)$ & $32,738(100.00)$ \\
\hline Mortality rate & 0.05 & 0.04 & 0.03 & 0.04 \\
\hline Mean length of stay, d & 5.22 & 5.41 & 5.00 & 5.18 \\
\hline Mean charge, \$ & $38,101.53$ & $39,401.45$ & $41,222.76$ & $40,099.73$ \\
\hline Mean cost, $\$$ & $12,964.16$ & $13,579.89$ & $12,647.02$ & $13,041.83$ \\
\hline \multicolumn{5}{|c|}{ Women aged 18-44 y } \\
\hline \multicolumn{5}{|l|}{ White } \\
\hline n (\%) & $1,173,549(66.97)$ & $491,581(28.05)$ & $87,176(4.97)$ & $1,752,306(100.00)$ \\
\hline Mortality rate & 0.00 & 0.01 & 0.01 & 0.00 \\
\hline Mean length of stay, $d$ & 3.45 & 4.63 & 4.88 & 3.85 \\
\hline Mean charge, $\$$ & $26,383.05$ & $26,096.09$ & $30,033.49$ & $26,483.96$ \\
\hline Mean cost, $\$$ & $8,282.15$ & $8,209.02$ & $9,332.88$ & $8,313.86$ \\
\hline \multicolumn{5}{|l|}{ Black } \\
\hline n (\%) & $329,648(57.58)$ & $187,273(32.71)$ & $55,613(9.71)$ & $572,533(100.00)$ \\
\hline Mortality rate & 0.00 & 0.01 & 0.01 & 0.01 \\
\hline Mean length of stay, $d$ & 4.02 & 5.17 & 5.43 & 4.53 \\
\hline Mean charge, $\$$ & $28,225.17$ & $32,018.64$ & $33,067.90$ & $29,937.95$ \\
\hline Mean cost, $\$$ & $8,090.41$ & $9,097.29$ & $9,832.92$ & $8,589.50$ \\
\hline \multicolumn{5}{|l|}{ Hispanic } \\
\hline $\mathrm{n}(\%)$ & $262,448(73.93)$ & $77,565(21.85)$ & $14,985(4.22)$ & $354,998(100.00)$ \\
\hline Mortality rate & 0.00 & 0.01 & 0.01 & 0.01 \\
\hline Mean length of stay, $d$ & 3.54 & 5.02 & 5.36 & 3.94 \\
\hline Mean charge, $\$$ & $32,328.97$ & $38,678.41$ & $43,519.81$ & $34,183.84$ \\
\hline Mean cost, \$ & $8,518.59$ & $9,898.87$ & $10,984.73$ & $8,923.22$ \\
\hline \multicolumn{5}{|l|}{ Asian/Pacific Islander } \\
\hline n (\%) & $38,944(75.83)$ & $10,754(20.94)$ & $1,660(3.23)$ & $51,358(100.00)$ \\
\hline Mortality rate & 0.01 & 0.01 & 0.01 & 0.01 \\
\hline Mean length of stay, $d$ & 3.84 & 5.93 & 6.40 & 4.36 \\
\hline Mean charge, $\$$ & $36,230.32$ & $51,448.43$ & $55,025.04$ & $39,983.62$ \\
\hline Mean cost, $\$$ & $9,762.20$ & $13,694.84$ & $14,330.96$ & $10,723.20$ \\
\hline
\end{tabular}


Preventing Chronic Disease I Hospital Utilization, Costs, and Mortality for Adults With... Page 15 of 20

\begin{tabular}{|c|c|c|c|c|}
\hline \multirow[b]{2}{*}{ Discharge Characteristic } & \multicolumn{4}{|c|}{ No. of Chronic Conditions } \\
\hline & 0 to 1 & 2 to 3 & $\geq 4$ & All \\
\hline $\mathrm{n}(\%)$ & $16,149(62.46)$ & $7,869(30.43)$ & $1,838(7.11)$ & $25,856(100.00)$ \\
\hline Mortality rate & 0.01 & 0.01 & 0.01 & 0.01 \\
\hline Mean length of stay, d & 3.60 & 5.34 & 5.05 & 4.23 \\
\hline Mean charge, $\$$ & $24,601.77$ & $28,011.90$ & $28,250.57$ & $25,901.55$ \\
\hline Mean cost, \$ & $8,381.63$ & $9,448.44$ & $9,081.64$ & $8,756.74$ \\
\hline \multicolumn{5}{|c|}{ Women aged 45-64 y } \\
\hline \multicolumn{5}{|l|}{ White } \\
\hline n (\%) & $1,186,996(36.45)$ & $1,335,870(41.03)$ & $733,201(22.52)$ & $3,256,067(100.00)$ \\
\hline Mortality rate & 0.01 & 0.01 & 0.01 & 0.01 \\
\hline Mean length of stay, d & 4.13 & 4.84 & 5.12 & 4.65 \\
\hline Mean charge, $\$$ & $33,859.10$ & $35,445.57$ & $36,614.66$ & $35,129.71$ \\
\hline Mean cost, \$ & $10,554.25$ & $11,064.03$ & $11,351.18$ & $10,942.63$ \\
\hline \multicolumn{5}{|l|}{ Black } \\
\hline n (\%) & $198,781(24.01)$ & $359,803(43.45)$ & $269,462(32.54)$ & $828,046(100.00)$ \\
\hline Mortality rate & 0.02 & 0.02 & 0.02 & 0.02 \\
\hline Mean length of stay, $d$ & 4.79 & 5.43 & 5.60 & 5.33 \\
\hline Mean charge, $\$$ & $36,747.47$ & $38,743.52$ & $38,706.02$ & $38,252.29$ \\
\hline Mean cost, \$ & $10,416.47$ & $10,908.23$ & $11,161.01$ & $10,872.44$ \\
\hline \multicolumn{5}{|l|}{ Hispanic } \\
\hline n (\%) & $150,184(36.90)$ & $162,767(39.99)$ & $94,051(23.11)$ & $407,002(100.00)$ \\
\hline Mortality rate & 0.01 & 0.01 & 0.02 & 0.01 \\
\hline Mean length of stay, d & 4.08 & 4.94 & 5.37 & 4.72 \\
\hline Mean charge, $\$$ & $38,043.64$ & $43,204.39$ & $46,865.87$ & $42,145.14$ \\
\hline Mean cost, $\$$ & $9,747.41$ & $10,866.26$ & $11,670.82$ & $10,639.10$ \\
\hline \multicolumn{5}{|l|}{ Asian/Pacific Islander } \\
\hline n (\%) & $34,958(42.26)$ & $32,206(38.93)$ & $15,562(18.81)$ & $82,725(100.00)$ \\
\hline Mortality rate & 0.02 & 0.02 & 0.02 & 0.02 \\
\hline Mean length of stay, $d$ & 4.25 & 5.23 & 5.60 & 4.88 \\
\hline Mean charge, $\$$ & $44,425.20$ & $52,586.01$ & $59,232.48$ & $50,306.06$ \\
\hline Mean cost, \$ & $11,829.17$ & $13,621.52$ & $15,001.78$ & $13,106.22$ \\
\hline \multicolumn{5}{|l|}{ Native American } \\
\hline $\mathrm{n}(\%)$ & $11,639(30.74)$ & $15,921(42.06)$ & $10,298(27.20)$ & $37,858(100.00)$ \\
\hline Mortality rate & 0.02 & 0.01 & 0.02 & 0.01 \\
\hline Mean length of stay, $d$ & 4.19 & 4.80 & 5.00 & 4.66 \\
\hline Mean charge, $\$$ & $30,139.27$ & $32,174.50$ & $34,456.78$ & $32,167.51$ \\
\hline Mean cost, \$ & $10,441.54$ & $10,864.81$ & $10,912.14$ & $10,747.29$ \\
\hline \multicolumn{5}{|c|}{ Women aged $\geq 65 y$} \\
\hline
\end{tabular}


Preventing Chronic Disease I Hospital Utilization, Costs, and Mortality for Adults With... Page 16 of 20

\begin{tabular}{|c|c|c|c|c|}
\hline \multirow[b]{2}{*}{ Discharge Characteristic } & \multicolumn{4}{|c|}{ No. of Chronic Conditions } \\
\hline & 0 to 1 & 2 to 3 & $\geq 4$ & All \\
\hline n (\%) & $829,817(13.71)$ & $2,456,096(40.58)$ & $2,766,399(45.71)$ & $6,052,312(100.00)$ \\
\hline Mortality rate & 0.04 & 0.03 & 0.04 & 0.04 \\
\hline Mean length of stay, d & 5.18 & 5.19 & 5.41 & 5.29 \\
\hline Mean charge, $\$$ & $35,414.11$ & $33,773.19$ & $33,429.61$ & $33,842.53$ \\
\hline Mean cost, $\$$ & $11,057.53$ & $10,506.00$ & $10,386.20$ & $10,527.33$ \\
\hline \multicolumn{5}{|l|}{ Black } \\
\hline n (\%) & $68,195(9.19)$ & $291,036(39.22)$ & $382,811(51.59)$ & $742,042(100.00)$ \\
\hline Mortality rate & 0.06 & 0.04 & 0.03 & 0.04 \\
\hline Mean length of stay, $d$ & 6.71 & 6.10 & 5.98 & 6.10 \\
\hline Mean charge, $\$$ & $48,248.46$ & $41,551.23$ & $38,663.57$ & $40,683.88$ \\
\hline Mean cost, \$ & $12,980.58$ & $11,515.06$ & $11,113.04$ & $11,443.54$ \\
\hline \multicolumn{5}{|l|}{ Hispanic } \\
\hline $\mathrm{n}(\%)$ & $69,704(14.14)$ & $206,336(41.86)$ & $216,909(44.00)$ & $492,949(100.00)$ \\
\hline Mortality rate & 0.04 & 0.03 & 0.03 & 0.03 \\
\hline Mean length of stay, $d$ & 5.14 & 5.35 & 5.83 & 5.53 \\
\hline Mean charge, $\$$ & $44,228.99$ & $45,217.73$ & $49,240.79$ & $46,840.52$ \\
\hline Mean cost, $\$$ & $10,717.20$ & $10,871.76$ & $11,856.34$ & $11,281.33$ \\
\hline \multicolumn{5}{|l|}{ Asian/Pacific Islander } \\
\hline n (\%) & $21,015(14.31)$ & $61,799(42.07)$ & $64,088(43.63)$ & $146,902(100.00)$ \\
\hline Mortality rate & 0.05 & 0.04 & 0.04 & 0.04 \\
\hline Mean length of stay, $d$ & 5.70 & 5.53 & 5.89 & 5.71 \\
\hline Mean charge, $\$$ & $54,013.97$ & $52,729.35$ & $59,779.75$ & $55,956.26$ \\
\hline Mean cost, \$ & $13,741.25$ & $12,850.96$ & $14,155.06$ & $13,542.71$ \\
\hline \multicolumn{5}{|l|}{ Native American } \\
\hline n (\%) & $6,502(15.77)$ & $16,773(40.67)$ & $17,962(43.56)$ & $41,236(100.00)$ \\
\hline Mortality rate & 0.05 & 0.03 & 0.03 & 0.03 \\
\hline Mean length of stay, d & 4.95 & 5.06 & 5.25 & 5.13 \\
\hline Mean charge, $\$$ & $30,440.56$ & $32,409.60$ & $34,475.43$ & $32,996.89$ \\
\hline Mean cost, $\$$ & $11,157.61$ & $11,059.78$ & $10,675.72$ & $10,908.19$ \\
\hline
\end{tabular}

Table 4. Five Most Prevalent Chronic Condition Dyads for US Adults With 2 or More Chronic Conditions, by Sex and Age, Nationwide Inpatient Sample, 2009

\begin{tabular}{|l|r|}
\hline Sex, Age, and Dyad & \%a \\
\hline \multicolumn{1}{|c|}{ Men } & \\
\hline $\mathbf{1 8 - 4 4}$ y (n= 1,044,459) & 23.9 \\
\hline Depression/substance abuse & 17.8 \\
\hline Hypertension/diabetes &
\end{tabular}


Preventing Chronic Disease I Hospital Utilization, Costs, and Mortality for Adults With... Page 17 of 20

\begin{tabular}{|c|c|}
\hline Sex, Age, and Dyad & $\%{ }^{a}$ \\
\hline Hyperlipidemia/hypertension & 15.1 \\
\hline Hypertension/substance abuse & 13.6 \\
\hline Hypertension/depression & 11.9 \\
\hline \multicolumn{2}{|l|}{$45-64$ y $(n=3,420,573)$} \\
\hline Hypertension/hyperlipidemia & 31.8 \\
\hline Hypertension/diabetes & 29.5 \\
\hline Hypertension/coronary artery disease & 26.1 \\
\hline Hyperlipidemia/coronary artery disease & 19.5 \\
\hline Diabetes/hyperlipidemia & 16.6 \\
\hline \multicolumn{2}{|l|}{$\geq 65$ y $(n=5,103,409)$} \\
\hline Hypertension/coronary artery disease & 37.1 \\
\hline Hypertension/hyperlipidemia & 34.2 \\
\hline Hypertension/cardiac arrhythmia & 28.8 \\
\hline Hypertension/diabetes & 27.9 \\
\hline Hyperlipidemia/coronary artery disease & 24.9 \\
\hline \multicolumn{2}{|c|}{ Women } \\
\hline \multicolumn{2}{|l|}{$18-44$ y $(n=987,310)$} \\
\hline Depression/substance abuse & 22.0 \\
\hline Hypertension/diabetes & 18.6 \\
\hline Hypertension/depression & 15.7 \\
\hline Depression/asthma & 12.5 \\
\hline Hyperlipidemia/hypertension & 11.3 \\
\hline \multicolumn{2}{|l|}{$45-64$ y $(n=3,150,679)$} \\
\hline Hypertension/diabetes & 31.3 \\
\hline Hyperlipidemia/hypertension & 28.3 \\
\hline Hypertension/depression & 18.0 \\
\hline Hyperlipidemia/diabetes & 16.7 \\
\hline Hypertension/coronary artery disease & 16.0 \\
\hline \multicolumn{2}{|l|}{$\geq 65$ y $(n=6,618,736)$} \\
\hline Hypertension/hyperlipidemia & 32.6 \\
\hline Hypertension/coronary artery disease & 26.9 \\
\hline Hypertension/diabetes & 26.7 \\
\hline Hypertension/cardiac arrhythmia & 25.9 \\
\hline Hypertension/congestive heart failure & 19.2 \\
\hline
\end{tabular}

a Percentage does not total $100 \%$ because the list presents only the top-ranked disease groupings. 
Preventing Chronic Disease I Hospital Utilization, Costs, and Mortality for Adults With... Page 18 of 20

Table 5. Five Most Prevalent Chronic Condition Triads for US Adults With 3 or More Chronic Conditions, by Sex and Age, Nationwide Inpatient Sample, 2009

\begin{tabular}{|c|c|}
\hline Sex, Age, and Triad & $\%{ }^{a}$ \\
\hline \multicolumn{2}{|c|}{ Men } \\
\hline \multicolumn{2}{|l|}{$18-44$ y $(n=481,305)$} \\
\hline Diabetes/hyperlipidemia/hypertension & 14.3 \\
\hline Hypertension/depression/substance abuse & 10.7 \\
\hline Diabetes/hyperlipidemia/chronic kidney disease & 10.0 \\
\hline Hypertension/hyperlipidemia/coronary artery disease & 9.2 \\
\hline Diabetes/hypertension/depression & 6.8 \\
\hline \multicolumn{2}{|l|}{$45-64$ y $(n=2,359,061)$} \\
\hline Hypertension/hyperlipidemia/coronary artery disease & 22.7 \\
\hline Diabetes/hyperlipidemia/hypertension & 20.1 \\
\hline Diabetes/hypertension/coronary artery disease & 16.7 \\
\hline Hyperlipidemia/coronary artery disease/diabetes & 11.7 \\
\hline Diabetes/hypertension/chronic kidney disease & 11.0 \\
\hline \multicolumn{2}{|l|}{$\geq 65$ y $(n=4,123,675)$} \\
\hline Hypertension/hyperlipidemia/coronary artery disease & 25.1 \\
\hline Hypertension/coronary artery disease/cardiac arrhythmia & 20.3 \\
\hline Diabetes/hypertension/coronary artery disease & 18.2 \\
\hline Diabetes/hyperlipidemia/hypertension & 16.7 \\
\hline Hyperlipidemia/hypertension/cardiac arrhythmia & 16.2 \\
\hline \multicolumn{2}{|c|}{ Women } \\
\hline \multicolumn{2}{|l|}{$18-44$ y $(n=429,490)$} \\
\hline Diabetes/hyperlipidemia/hypertension & 13.6 \\
\hline Diabetes/hyperlipidemia/hypertension & 11.5 \\
\hline Diabetes/hypertension/depression & 9.9 \\
\hline Diabetes/hypertension/chronic kidney disease & 7.9 \\
\hline Hypertension/hyperlipidemia/depression & 7.7 \\
\hline \multicolumn{2}{|l|}{$45-64$ y $(n=2,075,306)$} \\
\hline Diabetes/hyperlipidemia/hypertension & 22.7 \\
\hline Hypertension/hyperlipidemia/coronary artery disease & 21.0 \\
\hline Diabetes/hypertension/coronary artery disease & 13.6 \\
\hline Hypertension/hyperlipidemia/depression & 13.2 \\
\hline Diabetes/hypertension/chronic kidney disease & 10.4 \\
\hline \multicolumn{2}{|l|}{$\geq 65$ y $(n=5,208,808)$} \\
\hline Hypertension/hyperlipidemia/coronary artery disease & 16.9 \\
\hline Diabetes/hyperlipidemia/hypertension & 15.2 \\
\hline Hypertension/coronary artery disease/cardiac arrhythmia & 13.5 \\
\hline Diabetes/hypertension/coronary artery disease & 13.2 \\
\hline
\end{tabular}


Preventing Chronic Disease I Hospital Utilization, Costs, and Mortality for Adults With... Page 19 of 20

\begin{tabular}{|l|r|}
\hline Sex, Age, and Triad & \%a \\
\hline Hyperlipidemia/hypertension/cardiac arrhythmia & 13.0 \\
\hline
\end{tabular}

a Percentage does not total $100 \%$ because the list presents only the top-ranked disease groupings.

\section{Appendix. Coding for Selected Chronic Conditions}

\begin{tabular}{|c|c|}
\hline Condition & $\begin{array}{l}\text { CCS Category or } \\
\text { Cluster }\end{array}$ \\
\hline Hypertension & 98,99 \\
\hline Hyperlipidemia & 53 \\
\hline Congestive heart failure & 108 \\
\hline $\begin{array}{l}\text { Coronary artery disease (CAD) (includes acute myocardial infarction, which indicates chronic } \\
\text { underlying CAD) }\end{array}$ & 100,101 \\
\hline Diabetes & 49,50 \\
\hline Stroke (includes acute stroke but indicates underlying cerebrovascular disease) & $109-112$ \\
\hline Cardiac arrhythmias & 105,106 \\
\hline Arthritis & 202,203 \\
\hline Cancer & $11-43$ \\
\hline Depression & 657 \\
\hline Dementia (includes Alzheimer's and other senile dementias) & 653 \\
\hline Substance abuse disorders & 660,661 \\
\hline Chronic obstructive pulmonary disease & 127 \\
\hline Asthma & 128 \\
\hline Chronic kidney disease & 156,158 \\
\hline HIV & 5 \\
\hline Hepatitis & 6 \\
\hline Autism spectrum disorder & $\begin{array}{r}\text { ICD-9-CM 29900, } \\
29901\end{array}$ \\
\hline Schizophrenia & 659 \\
\hline Osteoporosis & 206 \\
\hline
\end{tabular}

The opinions expressed by authors contributing to this journal do not necessarily reflect the opinions of the U.S. Department of Health and Human Services, the Public Health Service, the Centers for Disease Control and Prevention, or the authors' affiliated institutions.

The RIS file format is a text file containing bibliographic citations. These files are best suited for import into bibliographic management applications such as EndNote $\mathbf{w}^{\mathrm{s}}$, Reference Manager download is available at each application's web site.

For Questions About This Article Contact pcdeditor@cdc.gov

Page last reviewed: May 30, 2013

Page last updated: May 30, 2013

Content source: National Center for Chronic Disease Prevention and Health Promotion

Centers for Disease Control and Prevention 1600 Clifton Rd. Atlanta, GA 30333, USA

80o-CDC-INFO (800-232-4636) TTY: (888) 232-6348 - Contact CDC-INFO 